\title{
Sleep and daytime sleepiness in methylphenidate medicated and un-medicated children with attention-deficit/hyperactivity disorder (ADHD)
}

\author{
K Cockcroft', J Ashwal', A Bentley² \\ 1Department of Psychology, School of Human and Community Development (Psychology), University of the Witwatersrand, \\ Johannesburg, South Africa \\ 2Wits Dial-a-Bed Sleep Laboratory, Brain Function Research Group, School of Physiology, University of the Witwatersrand, \\ Johannesburg, South Africa
}

\begin{abstract}
Objective: Excessive daytime sleepiness due to any cause can result in various symptoms similar to those used for the diagnosis of attention deficit/hyperactivity disorder (ADHD). A common treatment for children diagnosed with ADHD is methylphenidate which is also used to treat excessive daytime sleepiness. This paper reports on a study which compared the perceived levels of daytime sleepiness and prevalence of sleep disorders in medicated and un-medicated children with ADHD. Method: The sample consisted of two matched groups of children who had been formally diagnosed with ADHD. One group ( $\mathrm{n}=12$ ) was taking immediate release methylphenidate twice daily, while the other group ( $\mathrm{n}=1 \mathrm{l}$ ) had never, and were not currently, taking any medication. The two groups, as well as their parents, rated their levels of daytime sleepiness at three points in a single day. Results: Significantly higher levels of daytime sleepiness were reported by the parents of the un-medicated children between the hours of 13:00 and 15:00, compared to the medicated children. The medicated children became increasingly sleepier from the first to the second measurement in both the morning and afternoon. There was no significant difference in the number of sleep disorders/disruptions reported by the parents of either group. Conclusion: In a group of children with ADHD taking methylphenidate, there was a significant increase in sleepiness a few hours after taking the medication, which may then have a significant impact on their learning. The data also imply that part of the mechanism of action of methylphenidate effects in these children may be by reduction of daytime sleepiness.
\end{abstract}

Keywords: Attention Deficit Disorder with hyperactivity; Methylphenidate; Disorders of excessive somnolence

Received: $26-08-2008$

Accepted: 04-1 1-2008

\section{Introduction}

There is growing concern over increased daytime sleepiness among children and adolescents. ${ }^{1}$ Excessive daytime sleepiness stemming from sleep deprivation can result in mood changes, inattention, delayed reaction time,

Correspondence:
Prof. K Cockcroft
Department of Psychology, School of Human and Community
Development (Psychology), University of the Witwatersrand
PO WITS 2050, South Africa
email: kate.cockcroft@wits.ac.za

impaired vigilance, decreased motivation, hyperactivity, aggressive behavior and impulsivity. The symptoms displayed by sleepy children are similar to those used as diagnostic criteria for attention-deficit/hyperactivity disorder (ADHD), the most common childhood disorder, afflicting between 5 and 10 percent of school age children. ${ }^{2,3}$ Although sleep disruptions appear to be common in children with ADHD, no reference is made to sleep behaviour in the current diagnostic criteria (DSM-IV TR), as the aetiology of such behaviour is not clearly understood. ${ }^{4}$

There is some debate about the relationship between 
ADHD, sleep disorders and daytime sleepiness. Suggestions include that ADHD causes sleep disorders ${ }^{5}$, that sleep disorders and the resultant daytime sleepiness could either cause or exacerbate ADHD-like symptoms ${ }^{6,7,8}$ and that stimulant medications, the most widely prescribed treatment for $\mathrm{ADHD}$, could exacerbate or cause sleep disorders, or in fact are effective because they treat sleepiness during the day. ${ }^{5}$ These differing views stem from the inconsistent research findings in the field.

Many sleep disorders have been associated with ADHD, particularly those characterised by a difficulty in maintaining sleep, such as periodic limb movements in sleep, restless leg syndrome, sleep-disordered breathing and snoring or sleep apnea, all of which may result in insufficient or poor quality sleep. 5,6,7,9,10,11,12 Difficulties around "sleep-wake" transitions, such as bed-time refusal, delayed sleep-onset and early awakenings are also common in children with ADHD. In addition, children with ADHD show a longer duration of sleep with poorer sleep efficiency, twice as many nocturnal awakenings, reduced percentages of REM sleep and increased REM latencies compared to children without ADHD. ${ }^{13,14,15,16,17}$

As far as symptoms are concerned, persistent lack of sleep, for any of the reasons given above, may have farreaching negative consequences for the child, such as impaired prefrontal cortex functioning, resulting in poorer functioning in the domains of attention, learning, memory, language, visual-spatial skills, executive functioning and emotions. ${ }^{13,14,18,19,20}$ Poor sleep in children has also been associated with significantly greater anxiety, daytime fatigue, nocturnal enuresis, fear of sleeping alone, depression, poor school achievement, learning problems, behavioural problems, inattention, disorientation, speech deterioration, poor judgement, poor impulse control, irritability, confusion and hyperactivity. ${ }^{21,22}$

An additional complication in determining the nature of the above relationship is that central nervous system stimulants, the most common treatment of $\mathrm{ADHD}$, may also lead to changes in a child's sleep or worsen pre-existing disorders. ${ }^{23,24}$ Many of the putative sleep problems associated with "sleep-wake" transitions in children with ADHD have been attributed to the use of stimulants particularly when taken later in the day. Studies evaluating the effects of stimulants on the sleep of children with ADHD have yielded conflicting results, which may be due to sampling and/or methodological issues. For example, O'Brien et al ${ }^{18}$ found that methylphenidate used for the behavioural management of ADHD does not appear to be associated with any detrimental effects on either subjective or objective measures of sleep. A limitation of this study was that the authors were unable to perform rigorous assessments for ADHD. On the other hand, Barkley et $\mathrm{al}^{25}$ found significantly more parental reports of insomnia in children with ADHD who were administered stimulant medication than in children who were un-medicated. Medication schedule, type and dosage may influence sleep outcome measures, making it difficult to compare studies. ${ }^{26}$ In addition, methylphenidate, the stimulant most often used in the treatment of ADHD symptoms, is used in sleep medicine to reverse excessive daytime sleepiness, particularly in narcolepsy. ${ }^{27}$ Thus, methylphenidate may be both causing difficulties in falling asleep at night as well as treating the effects of such difficulties the following day.

This exploratory study investigated whether treatment with methylphenidate had an effect on daytime sleepiness in children with ADHD and whether these changes could be noticed by parents and/or the children themselves. Of further interest was whether daytime sleepiness could be linked to concomitant sleep disorders in this population.

\section{Methods}

Sample

A total of 23 children (16 boys and 7 girls) in Grades one through six (ranging in age from 6.4 years to 12.7 years), from three South African Gauteng Department of Education (GDE) remedial primary schools participated in this study. This particular age group was selected because the primary school years are typically when ADHD is first diagnosed. The school psychologist in each school was approached and requested to deliver letters to the parents of all children who had been diagnosed with ADHD, inviting them to participate in the study. This yielded positive responses from 30 parents, 11 with un-medicated children with ADHD and 19 with medicated children. All were included in the study, although 7 children from the medicated group were omitted from the final analyses as they were either taking medication other than methylphenidate, or matches with un-medicated children could not be found for them, or they had concurrent medical conditions which may have served as extraneous variables. The groups were matched on the basis of age, grade, gender and ethnicity. The un-medicated group ( $\mathrm{n}=11$ ) had never, and were not currently, using any prescription medication, while the medicated group $(n=12)$ were taking only immediate release methylphenidate twice a day, once in the morning and once in the afternoon. All of the participants had been diagnosed with ADHD by a psychologist, in all cases by using both the Conner's Rating Scale and DSM IV TR diagnostic criteria.

\section{Instruments}

This study used two measurements, namely a parental questionnaire and the Wits Faces Sleepiness Scale, a pictorial measure of daytime sleepiness. ${ }^{28}$

The parental questionnaire, which consisted of 12 openended questions, was formulated by the authors and completed by the parents. It provided information regarding each child's sleep habits, sleep patterns (total sleep time, sleep latency, night time arousals, daytime naps and comments regarding daytime sleepiness in the children from appropriate adults, as well as symptoms and signs of the common sleep disorders as delineated in the DSM-IV TR). ${ }^{4}$ The questionnaire also included a visual analogue scale anchored at "not at all sleepy" to "very sleepy" on which the parents were requested to rate their children's general levels of sleepiness on an average day, at two points in the day, namely between the hours of 13:00 and 15:00, and between 17:00 and 19:00.

The children were all given the Wits Faces Sleepiness Scale ${ }^{28}$ to complete. This is a subjective, pictorial scale designed specifically for children which consists of five cartoon faces depicting increasing levels of sleepiness. 


\section{Procedure}

The children rated their level of sleepiness by choosing one of the faces on the Wits Faces Sleepiness Scale that most closely corresponded with their present state. The Scale was administered on two separate occasions during the course of a single school day - once in the morning at 8:30 am, prior to any breaks, and once in the afternoon at 13h00, after the lunch break. The Scale was administered on the same day and at the same times for all participants. All of the children in the medicated group had taken their usual stimulants in the morning before the first administration of the Scale, but completed the second administration of the Scale before their second dose of stimulants was taken.

\section{Ethics}

The project was approved by the Committee for Research on Human Subjects of the University of the Witwatersrand. The data were treated confidentially, and once the test results had been matched, they were coded to ensure anonymity. The schools and parents obtained written and oral feedback regarding group performance only.

\section{Statistical analysis}

Demographic data were compared between groups using an independent pairs t-test. Differences between sleepiness scales at the two different times of the day were compared using a matched pairs t-test, and differences between groups (medicated and un-medicated) were compared using an independent pairs t-test. A Fishers exact test was used to compare the number of sleep disorders between groups as well as the manner of morning awakening, as obtained from the parental questionnaire. Data from the parental visual analogue scale was subjected to arcsine transformation prior and post analysis to produce parametric data.

\section{Results}

There was no significant difference between the medicated and un-medicated groups in terms of age, gender and grade. In addition, there were no significant differences between the medicated and un-medicated groups for time of going to bed, sleep latency, awakening time, prevalence of daytime naps, manner of morning awakening or comments on child's daytime sleepiness by the child's teacher, doctor or therapist.

The prevalence of various sleep disorders, as reported by the parents, in the two groups of children is presented in Table I.

\section{Table I: Percentage of sleep disturbances by parental report in medicated $(n=12)$ and un-medicated children $(n=11)$ with ADHD}

\begin{tabular}{|l|l|l|}
\hline Type of sleep disturbance & Medicated group \% & Un-medicated group \% \\
\hline Snoring & 16 & 0 \\
Non-breathing/gasping & 8 & 0 \\
Leg movements & 50 & 36 \\
Sleep walking & 25 & 9 \\
Nightmares & 42 & 36 \\
Talking in sleep & 16 & 18 \\
Various sleep positions & 8 & 0 \\
Restlessness & 8 & 0 \\
Enuresis & 16 & 0 \\
\hline
\end{tabular}

\begin{tabular}{|c|c|c|c|c|}
\hline Time of day & $\begin{array}{l}\text { Medicated } \\
\text { group }\end{array}$ & $\begin{array}{l}\text { Un-medicated } \\
\text { group }\end{array}$ & $\begin{array}{l}\text { Parents of } \\
\text { medicated } \\
\text { group }\end{array}$ & $\begin{array}{l}\text { Parents of } \\
\text { un-medicated } \\
\text { group }\end{array}$ \\
\hline $\begin{array}{l}8: 00 \\
13: 00-15: 00 \\
17: 00-19: 00\end{array}$ & $\begin{array}{l}0.31 \\
1.39^{\star} \\
-\end{array}$ & $\begin{array}{l}0.55 \\
0.87 * \\
-\end{array}$ & $\begin{array}{l}- \\
0.10 \# \\
1.08^{\star}\end{array}$ & $\begin{array}{l}- \\
0.98 \text { \# } \\
0.87 *\end{array}$ \\
\hline \multicolumn{5}{|c|}{$\begin{array}{l}{ }^{*} p<0.05 \text { when compared to earlier time in both cases (matched pairs } \\
\text { t-test). } \\
\# p<0.05 \text { when compared to un-medicated children at the same time } \\
\text { period (independent t-test). } \\
\text { - indicates no data obtained for that time period }\end{array}$} \\
\hline
\end{tabular}

Although the medicated group had only 1 out of 12 children with no sleep disorder as compared to 6 out of 11 unmedicated children without a sleep disorder, a Fisher's exact test yielded a nearly, but essentially not statistically significant difference ( $p=0.06)$, between the two groups. Of particular interest is the high prevalence of leg movements and nightmares in both groups. Snoring, apnea/ gasping, restlessness, frequent changes in sleep position and enuresis were only found in the medicated group.

When the medicated and un-medicated groups were separated in terms of presence or absence of sleep disorder, there was also no significant difference in their daytime ratings of sleepiness, whether by self or parental rating. For the latter analysis, leg movements and snoring were regarded as the only significant sleep disorders, since the others are unlikely to cause significant daytime sleepiness. Nine children presented with more than one sleep disorder.

There were no significant differences when comparing the perceived levels of daytime sleepiness, as expressed by the children in each group, at either the first or second rating of the day. There was, however, a significant increase in perceived daytime sleepiness in the medicated group $(t=-2.59 ; d f=8 ; p<0.05)$, but not in the un-medicated group, between 08:30 and 13:00 (Table II).

The parents of the un-medicated group perceived their children as having significantly higher levels of daytime sleepiness between the hours of 13:00 and 15:00 ( $t=2.77$; df $=9 ; \mathrm{p}<0.05)$ than the parents of the medicated group. Very few of the parents of the medicated children rated their child as sleepy at this time. No significant differences between the groups were evident for the parents' ratings of their children's sleepiness for the time period from 17:00 to 19:00. The parents of the un-medicated children did not perceive their children as significantly more sleepy as the day progressed, but the parents of the medicated children did $(t=2.45 ; \mathrm{df}=8 ; \mathrm{p}<0.05)$.

\section{Discussion}

This study formed a preliminary investigation into whether children diagnosed with ADHD and who are un-medicated, had higher levels of sleep disorders and daytime sleepiness when compared to children with $\mathrm{ADHD}$, who are on stimulant medication. No difference in either sleep length or 
disturbances or prevalence of sleep disorders was found. The children on medication themselves were aware of becoming significantly sleepier as the morning progressed, but at no time was their sleepiness higher than the unmedicated children. The medicated children, according to their parents, also became sleepier over the course of the afternoon. The un-medicated group were sleepier than the medicated group during the period of 13:00 -15:00 based on parental report.

Methylphenidate, the stimulant taken by all of the medicated children, blocks the reuptake, and increases release of, dopamine and norepinephrine from presynaptic vesicles into the synapse. The increases in extracellular dopamine and norepinephrine cause sympathomimetic effects resulting not only in increased performance on tasks requiring attention and concentration, but also a decreased need for sleep and decreased awareness of fatigue. Administering short-acting stimulants twice daily, as in the medicated sample, once in the morning and once just after lunch, may result in good coverage during the day but a lack of coverage in the evening hours when the child needs to be able to concentrate and interact in social and family situations. This may be preferable to additional late afternoon doses of stimulants which may adversely affect sleep, either due to a direct drug effect or due to a secondary "rebound" effect as the medication wears off. 1

Apart from actual sleep disorders there were no differences between the two groups of children in their bedtime behaviour. The results of studies investigating differences between the sleep behaviour of medicated and un-medicated children with ADHD have been contradictory. $5,18,22,26,28,29,30$ One of the few that focused on daytime sleepiness, as opposed to sleep disorders, found that un-medicated children with ADHD seem to have a diurnal variation in daytime alertness, with a dip around, or just before, lunch as seen in the children in our study.17

In general, very few studies reporting on levels of daytime sleepiness are available, but there are many that indicate parentally reported trouble sleeping or insomnia (both sleep onset and maintenance) in stimulant medicated children with ADHD, as opposed to un-medicated children with $\mathrm{ADHD}^{5}$, which would presumably influence daytime sleepiness. In the current study, there was no significant difference in the number of sleep disorders reported by the parents of the medicated and the un-medicated children, which corroborates results obtained with objective measures of daytime sleepiness. ${ }^{18}$

Subjective reports of sleep in children with ADHD have primarily been collected using parental reports of the type used in the current study. The current study is original in that it appears to be the first one in which children were asked to rate their own levels of daytime sleepiness which were then compared to their parent's reports. The medicated children reported low levels of sleepiness soon after taking their medication both by self (in the early morning) and parental report (in the early afternoon). Their level of sleepiness, however, increased by the second measurement on both occasions which was when the level of stimulant had decreased. This resulted in the loss of the normal circadian changes in sleepiness in the early afternoon as evidenced in the un-medicated group. Thus in our group of medicated children it appeared as though the methylphenidate was in fact reducing daytime sleepiness as part of its mechanism of action.

As reported earlier, previous studies that investigated the effects of stimulants on sleep have produced mixed results and it is difficult to ascertain from these studies whether the sleep disturbances resulted from the ADHD or from the stimulant medication. Sleep disturbances in children with ADHD could be related to the neurobehavioural abnormality itself or could result from over-alertness and nervousness, which affect both sleep and behaviour. Based on a parent questionnaire, Stein (1999) reported frequent night-time wakening in $25 \%$ of children with ADHD on stimulant medication compared with $9 \%$ of un-medicated children. However, O'Brien et al. (2003) found that parents of $\mathrm{ADHD}$ children, regardless of their medication status, were more likely to report sleep problems than were parents of controls, but that these reports were not confirmed by objective sleep assessments, such as PSG evaluations.

There was no significant difference between the medicated and un-medicated groups in terms of parentally reported sleep disorders. This was surprising given the large numerical difference between the groups, but is probably entirely due to low numbers in each sample. Of particular interest is the high prevalence of leg movements and nightmares in both groups. These are sleep disorders that are characterized by a difficulty in maintaining sleep and are thus associated with daytime sleepiness but the presence of the sleep disorder would not be substantially changed by administration of methylphenidate during the day. ${ }^{6}$ They have also been found to be more prevalent in children with ADHD. ${ }^{7}$ Equally important was the lack of correlation between daytime sleepiness and sleep disorders in both groups of children but the expected daytime sleepiness may be masked by treatment with methylphenidate.

\section{Conclusion}

In conclusion, despite the limitations associated with using a small sample, this study identified some valuable areas for consideration when dealing with children who have ADHD. The children with ADHD who were being treated with stimulants demonstrated significantly higher levels of subjectively measured daytime somnolence six hours after taking their stimulant medication both in the morning and the afternoon, suggesting that these children are likely to have considerably lowered alertness around these times, which would impact on their learning ability. In the early afternoon, the parents of the medicated group rated their children as less sleepy than did the parents of the unmedicated group, probably as a result of the stimulant medications being effective at that time.

While this study did not attempt to address the nature of the relationship between ADHD and sleep disorders, the data indicates a relatively high level of sleepiness in children with ADHD and a significant effect of the stimulant medication in reducing that level of sleepiness for the duration of the action of the medication. Due to the potential negative effects of disturbed sleep on daytime functioning, it is highly recommended that school aged children 
diagnosed with ADHD be routinely screened for daytime sleepiness. In this way the symptoms of problem sleepiness can be identified and appropriate management thereof can be initiated, including educating the child and family about the potential negative effects of daytime sleepiness and sleep disorders and how they may contribute to or aggravate the behavioural manifestations of ADHD. ${ }^{6}$

\section{References}

1. Fallone G, Owens JA, Deane J. Sleepiness in children and adolescents: Clinical implications. Sleep Med Rev 2002; 6(4): 287306.

2. Gottlieb DJ, Vezina RM, Chase C, Lesko SM, Heeren TC, WeeseMayer DE et al. Symptoms of sleep disordered breathing in 5-year-old children are associated with sleepiness and problem behaviours. Pediatr 2003; 114(4): 870-7.

3. Tsal, Y, Shalev, L, Mevorach, C. The diversity of attention deficits in ADHD: The prevalence of four cognitive factors in ADHD versus controls. J Learn Dis 2005; 38(2): 142-157.

4. American Psychological Association Diagnostic and Statistical Manual of Mental Disorders. 4th Edition: APA, 2000: 430-5.

5. Cohen-Zion M, Ancoli-Israel S. Sleep in children with attentiondeficit/hyperactivity disorder ADHD: A review of naturalistic and stimulant intervention studies. Sleep Med Rev 2004; 8(5): 379-402.

6. Arman AR, Ersu R, Save D, Karadag B, Kraman G, Karabekiroglu $K$ et al. Symptoms of inattention and hyperactivity in children with habitual snoring: evidence from a community-based study in Istanbul. Child Care Health Dev 2005; 31 (6): 707-17.

7. Chervin RD, Dillon JE, Bassetti C, Ganoczy DA, Pituch KJ. Symptoms of sleep disorders, inattention and hyperactivity in children. Sleep 1997; 20: 1185-92

8. Corkum P, Tannock R, Moldofsky H. Sleep disturbances in children with attention-deficit/hyperactivity disorder. J Am Acad Child Adolesc Psych 1998; 37(6): 637-46.

9. Konofal E, Lecendreux M, Bouvard MP, Mouren-Simeoni MC. High levels of nocturnal activity in children with attention-deficit hyperactivity disorder: A video analysis. Psychiatry Clin Neurosci 2002; 55(2): 97-103

10. Kooij JJ, Middelkoop HA, van Gils K, Buitelaar JK. The effect of stimulants on nocturnal motor activity and sleep quality in adults with ADHD: an open-label case-control study. J Clin Psychiatry 2001; 62: 952-6.

11. Marcotte AC, Thatcher PV, Butters M, Bortz, J, Acebo C, Carskadon MA. Parental report of sleep problems in children with attentional and learning disorders. J Dev Behav Pediatr 1998; 19(3): 178-86.

12. Smedje H, Broman JE, Hetta J. Associations between disturbed sleep and behavioral difficulties in 635 children aged six to eight years: A study based on parent's perceptions. Eur Child Adolesc Psychiatry 2001; 10: 1-9.

13. Andreou G, Karapetsas A, Agapiou P, Gourgoulianis K. Verbal intelligence and sleep disorders in children with ADHD. Percept Mot Skills 2003; 96(3): 1283-9.
14. Corkum P. Sleep Disturbances in children with Attention Deficit/Hyperactivity Disorder: A comparative study with healthy siblings. J Am Acad Child Adolesc Psych 1998; 37(6): 637-46.

15. Gruber R, Sadeh A, Raviv A. Instability of sleep patterns in children with attention-deficit/hyperactivity disorder. J Am Acad Child Adolesc Psych 2000; 39(4): 495-501.

16. Kaplan BJ, McNicol J, Conte RA, Moghadam HK Sleep disturbances in preschool aged hyperactive and non-hyperactive children. Pediatr 1987; 80(6): 839-44.

17. Lecendreux M, Konofal E, Bouvard M, Falissard B, Mouren-Simeon M. (2000). Sleep and alertness in children with ADHD. J Child Psychol Psychiatry, 41 (6), 802-12.

18. O'Brien LM, Ivanenko A, McLaughlin Crabtree V, Holbrook CR, Bruner JL, Klaus CJ et al. The effect of stimulants on sleep characteristics in children with attention deficit/hyperactivity disorder. Sleep Med 2003; 4: 309-16.

19. Horne JA. Human sleep loss and behaviour implications for the prefrontal cortex and psychiatric disorder. Br J Psychiatry 1993; 162: 413-19.

20. Siegel JM. The REM sleep-memory consolidation hypothesis. Science 2000; 294: 1058-63.

21. Sangal RB, Owens J, Allen AJ, Sutton,.. Schuh K, Kelsey D.Effects of Atomoxetine and Methylphenidate on sleep in children with ADHD. Sleep 2006; 29(12): 1573-85.

22. Ring A, Stein D, Barak, Y, Teicher A, Hadjez J, Elizur A et al. Sleep disturbances in children with attention-deficit/hyperactivity disorder: A comparative study with healthy siblings. J Learn Dis 1998; 1: 572-80

23. Swanson J. Compliance with stimulants for AttentionDeficit/Hyperactivity Disorder: Issues and approaches for improvement. CNS Drugs 2003; 17(2): 117-31.

24. Venter $A$. The medical management of attentiondeficit/hyperactivity disorder: spoilt for choice? S Afr Psychiatry Rev 2006; 9: 143-51

25. Barley RA, McMurray MD, Edelbrock CS, Robbins K. Side effects of methylphenidate in children with attention deficit hyperactivity disorder: a systemic, placebo-controlled evaluation. Pediatr 1990; 86:184-92

26. Efron D, Jarman F, Barker M. Side effects of methylphenidate and dexamphetamine in children with attention deficit/hyperactivity disorder: a double-blind, crossover trial. Pediatr 1997; 100: 662-6.

27. Billiard M, Bassetti C, Dauvilliers Y, Dolenc-Groselj L, Lammers GJ, Mayer $G$ et al. EFNS guidelines on management of narcolepsy. Eur J Neurol 2006; 13 (10): 1035-48.

28. Maldonado CC, Bentley AJ, Mitchell D. A pictorial sleepiness scale based on cartoon faces. Sleep 2004; 27(3): 541-7.

29. Stein MA. Unravelling sleep problems in treated and untreated children with ADHD. J Child Adolesc Psychopharmacol 1999; 9(3): 157-68.

30. Stein D, Pat-Horenczyk R, Blank S, Dagan Y, Barak Y, Gumpel TP. Sleep disturbances in adolescents with symptoms of AttentionDeficit/Hyperactivity Disorder. J Learn Dis 2002; 35(3): 268-76. 